\title{
Introducing the program Naaah (Na-O Anticorrelation And HB)
}

\author{
Eugenio Carretta ${ }^{1}$, Angela Bragaglia ${ }^{1}$, Raffaele Gratton ${ }^{2}$, \\ Franco Leone ${ }^{3}$, and Sara Lucatello ${ }^{2}$ \\ ${ }^{1}$ INAF-Osservatorio Astronomico di Bologna, via Ranzani 1, 40127 Bologna (Italy) \\ email: angela.bragaglia, eugenio.carretta @bo.astro.it \\ ${ }^{2}$ INAF-Osservatorio Astronomico di Padova \\ email: gratton, lucatello @pd.astro.it \\ ${ }^{3}$ INAF-Osservatorio Astronomico di Catania \\ email: fleone@ct.astro.it
}

\begin{abstract}
We present here the first results (on NGC 2808 and NGC 6752) of a program that studies the anticorrelation between $\mathrm{Na}$ and $\mathrm{O}$ in a sample of Galactic Globular Clusters, using GIRAFFE spectra obtained with UVES@VLT.
\end{abstract}

Keywords. Stars: abundances, stars: fundamental parameters, stars: Population II, globular clusters: general,globular clusters: individual (NGC 2808, NGC 6752)

\section{Our program: first results}

Are Globular Clusters (GCs) true example of Simple Stellar Population? Were the GCs able to sustain self-enrichment of metals? We started a ESO program (072.D-0507, 073.D-0211) with FLAMES to observe Na and O abundances in about 100 RGB stars in about 20 GCs with different HB morphologies and overall properties. We intend to probe the first billion years in the life of GCs, to test: (a) if a second generation of stars born from the ejecta of intermediate mass stars does exist in GCs and (b) if any correlation exists between the cluster properties, in particular the extension of the Horizontal Branch (HB), and the distribution of stars along the Na-O anticorrelation.

We used FLAMES@VLT in GIRAFFE-MEDUSA mode, observing with the two grat-

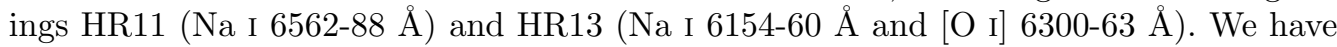
spectra for 130 and 151 stars respectively in NGC 2808 and NGC 6752; for 96 and 110 of these, respectively, we can measure both $\mathrm{O}$ and $\mathrm{Na}$ lines.

We derived the $\mathrm{T}_{\text {eff }}$ from the $V-K$ colour and the Alonso et al. (1999) calibration; for gravity we used the $\mathrm{T}_{\text {eff }}$ and the position in the colour-magnitude diagram; the microturbulent velocities were obtained by zeroeing the slope of the Fe I - expected line strenghts relation. We used the Kurucz (1993) atmospheric models. Abundances of O are from spectra corrected for telluric contamination, and $\mathrm{Na}$ abundances are corrected for departures from LTE (Gratton et al. 1999). Results of our analysis are shown in Fig. 1.

NGC 2808: Counting only detections, the bulk of stars is O-rich; however, following the overall $\mathrm{Na}-\mathrm{O}$ anticorrelation, a lot of super O-poor stars does appear, likely the counterparts of blue and extremely blue HB stars. There is a small difference $(0.023 \pm$ $0.012 \mathrm{dex})$ in the mean $[\mathrm{Fe} / \mathrm{H}]$ content of O-poor and O-rich stars. The difference is in agreement with what expected if O-depleted stars are also He-enriched, likely from the same polluting source (IM-AGB stars?). Because of a small differential reddening, no strong, definitive conclusions on the intrinsic Fe spread are possible. 

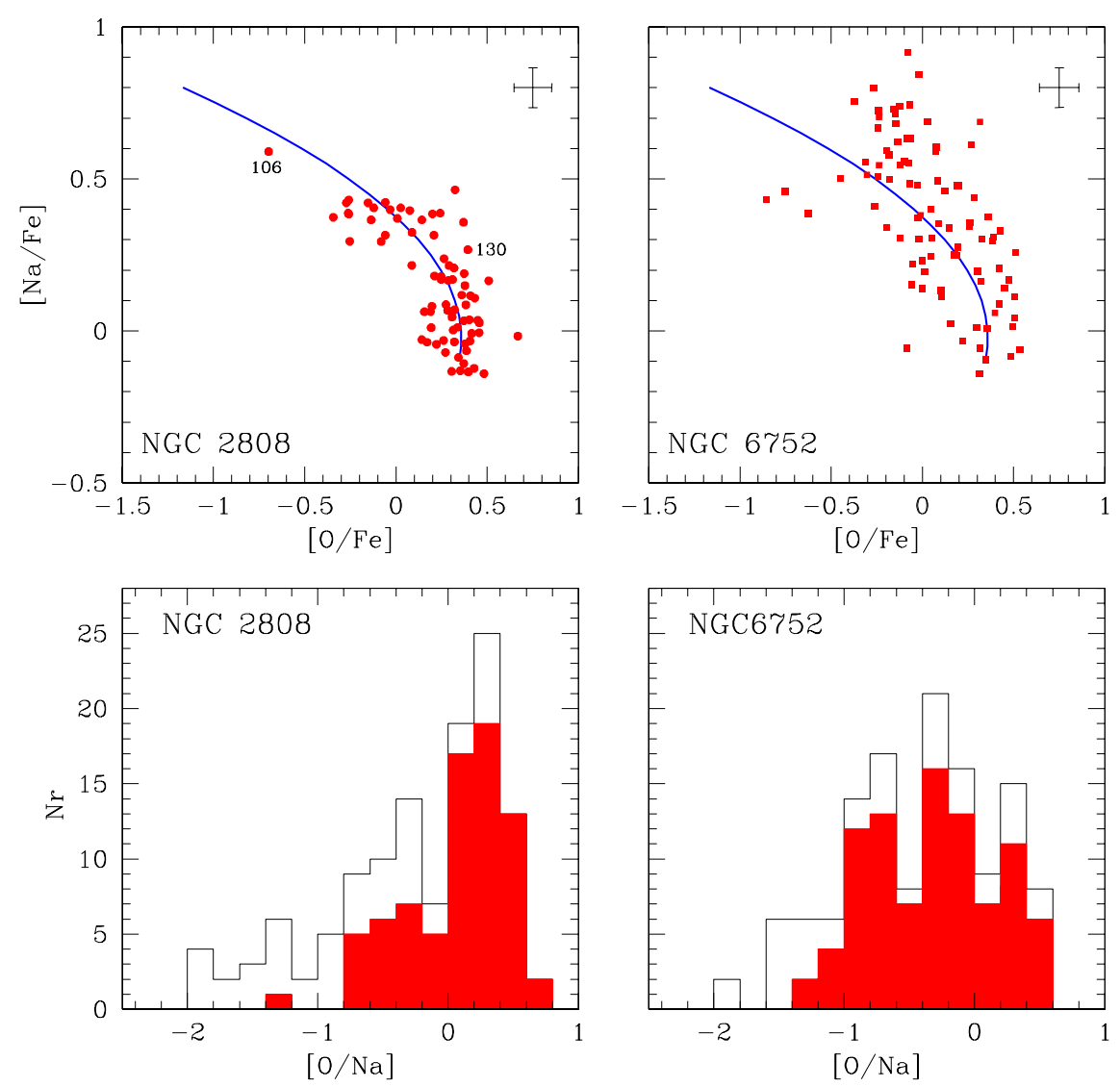

Figure 1. Upper panel, left: Na-O anticorrelation in NGC 2808. The blue line is the mean locus derived from $\sim 400$ RGB stars in 20 GCs from literature. Together with the most O-poor star detected, we indicate a star with very similar parameters but normal $\mathrm{O}$ abundance. Lower panel, left: Distribution of stars along the Na-O anticorrelation in NGC 2808. The filled histogram is for detections, and the open one is for objects with no HR 13 observations or undetectable $\mathrm{O}$ lines; in this case the predicted $[\mathrm{O} / \mathrm{Fe}]$ from the relation above is used. Right panels: the same, but for NGC 6752 ; in this case we plot also limits with the same symbols.

NGC 6752: The distribution of stars along the Na-O anticorrelation is different: there are more Na-poor stars compared to NGC 2808. The HB populated only in the blue part must be mostly due to the influence of the first parameter (metallicity) and maybe to the age. There is not much space for an intrinsic abundance spread in this cluster.

\section{Acknowledgements}

Other members of the team are: R. Buonanno, V. Caloi, R. Capuzzo-Dolcetta, R. Claudi, F. D'Antona, S. Desidera, P. François, G. Iannicola, G. James, M. Limongi, S. Moehler, E. Pancino, L. Pasquini, G. Piotto, A. Recio-Blanco, M. Zoccali.

\section{References}

Alonso, A., Arribas, S., \& Martinez-Roger, C. 1999, A\&SS 140, 261

Gratton, R.G., Carretta, E., Eriksson, K., Gustafsson, B. 1999, A 6 A 350, 955

Kurucz, R. 1993, CD-ROM No. 13, Smithsonian Astrophysical Observatory 